\title{
Malignant peripheral nerve sheath tumor in the anterior mediastinum: A case report
}

\author{
SATOSHI KOEZUKA ${ }^{1}$, YOSHINOBU HATA ${ }^{1}$, FUMITOMO SATO ${ }^{1}$, HAJIME OTSUKA ${ }^{1}$, \\ TAKASHI MAKINO $^{1}$, NAOBUMI TOCHIGI ${ }^{2}$ and AKIRA IYODA ${ }^{1}$ \\ ${ }^{1}$ Division of Chest Surgery; ${ }^{2}$ Department of Surgical Pathology, \\ School of Medicine, Toho University, Tokyo 143-8541, Japan
}

Received March 18, 2014; Accepted March 24, 2014

DOI: $10.3892 / \mathrm{mco} .2014 .343$

\begin{abstract}
Malignant peripheral nerve sheath tumors (MPNSTs) are rare neoplasms, accounting for only 5\% of all malignant soft-tissue sarcomas. This is the case report of a 28-year-old male patient with a MPNST located in the anterior mediastinum, originating from the phrenic nerve. The patient presented with an abnormal shadow on chest radiography and a $10-\mathrm{cm}$ mass in the right anterior mediastinum was identified on computed tomography and magnetic resonance imaging. The patient subsequently underwent surgical resection. The tumor originated from the right phrenic nerve and was tightly adherent to the middle and lower lobes of the right lung, the pericardium and the diaphragm. The tumor was completely resected, along with partial resection of the adherent tissues. The histological diagnosis was spindle cell sarcoma and the final diagnosis was MPNST of the anterior mediastinum, based on the characteristic microscopic appearance indicating the nervous origin of the tumor and on the intraoperative findings. The patient remains free of recurrence 1 year after surgery.
\end{abstract}

\section{Introduction}

Malignant peripheral nerve sheath tumors (MPNSTs) are rare neoplasms, accounting for only $5 \%$ of all malignant soft-tissue sarcomas $(1,2)$. The most common sites of occurrence are the torso, extremities and head and neck, whereas MPNSTs are extremely rarely located in the mediastinum (3). This is the case report of a patient with an MPNST in the anterior mediastinum, originating from the right phrenic nerve.

Correspondence to: Professor Akira Iyoda, Division of Chest Surgery, School of Medicine, Toho University, 6-11-1 Omori-nishi, Ota-ku, Tokyo 143-8541, Japan

E-mail: aiyoda@med.toho-u.ac.jp

Key words: malignant peripheral nerve sheath tumor, anterior mediastinum, mediastinal tumor

\section{Case report}

A 28-year-old man underwent screening chest radiography and was found to have an anterior mediastinal mass. There was no abnormal shadow on a chest radiograph obtained the previous year. The patient was asymptomatic, had no family history of neurofibromatosis type 1 (NF1) and his medical history included a right pneumothorax that was treated surgically, with pleurodesis, performed 3 years prior. The results of blood testing were unremarkable. A chest radiograph revealed a well-defined tumor, $10 \mathrm{~cm}$ in size, in the right anterior mediastinum (Fig. 1A). Contrast-enhanced computed tomography of the chest revealed a heterogeneous mass with rim enhancement; the mass compressed the diaphragm and the right atrium (Fig. 1B). Magnetic resonance imaging revealed an anterior mediastinal mass with heterogeneous high intensity on $\mathrm{T} 1$ and $\mathrm{T} 2$-weighted images. The area of high intensity persisted on fat saturation imaging and was therefore suspected to represent hemorrhage.

Considering the rapid growth of the lesion, its location and the possibility of malignancy, surgical resection of the tumor was recommended. Surgical access was obtained via a right posterolateral thoracotomy over the sixth rib. The tumor was located in the interlobular space, appearing to originate from the right phrenic nerve and was densely adherent to the middle and lower lobes of the right lung, the pericardium and the diaphragm. The tumor was completely resected and partial resection of the pericardium, right phrenic nerve, diaphragm and the middle and lower lobes of the right lung was also performed.

Macroscopically, the tumor was an encapsulated solid

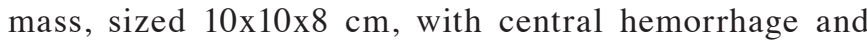
necrosis. Microscopically, the tumor exhibited highly cellular as well as loose regions, containing spindle-shaped tumor cells (Fig. 2A). Focal nuclear palisading was present (Fig. 2B), with high mitotic activity (30 mitotic figures per 10 high-power fields) and foci of necrosis. There was no tumor cell invasion of the neighboring organs.

On immunohistochemical analysis, the tumor cells were positive for vimentin, B-cell lymphoma 2 protein and cluster of differentiation (CD) 56, weakly positive for CD99 and negative for S-100 protein, synaptophysin, chromogranin A, h-caldesmon, $\alpha$-smooth muscle actin, desmin, calretinin, Wilms tumor protein 1 and CD34. Based on the these markers, the intraoperative findings and the characteristic microscopic 


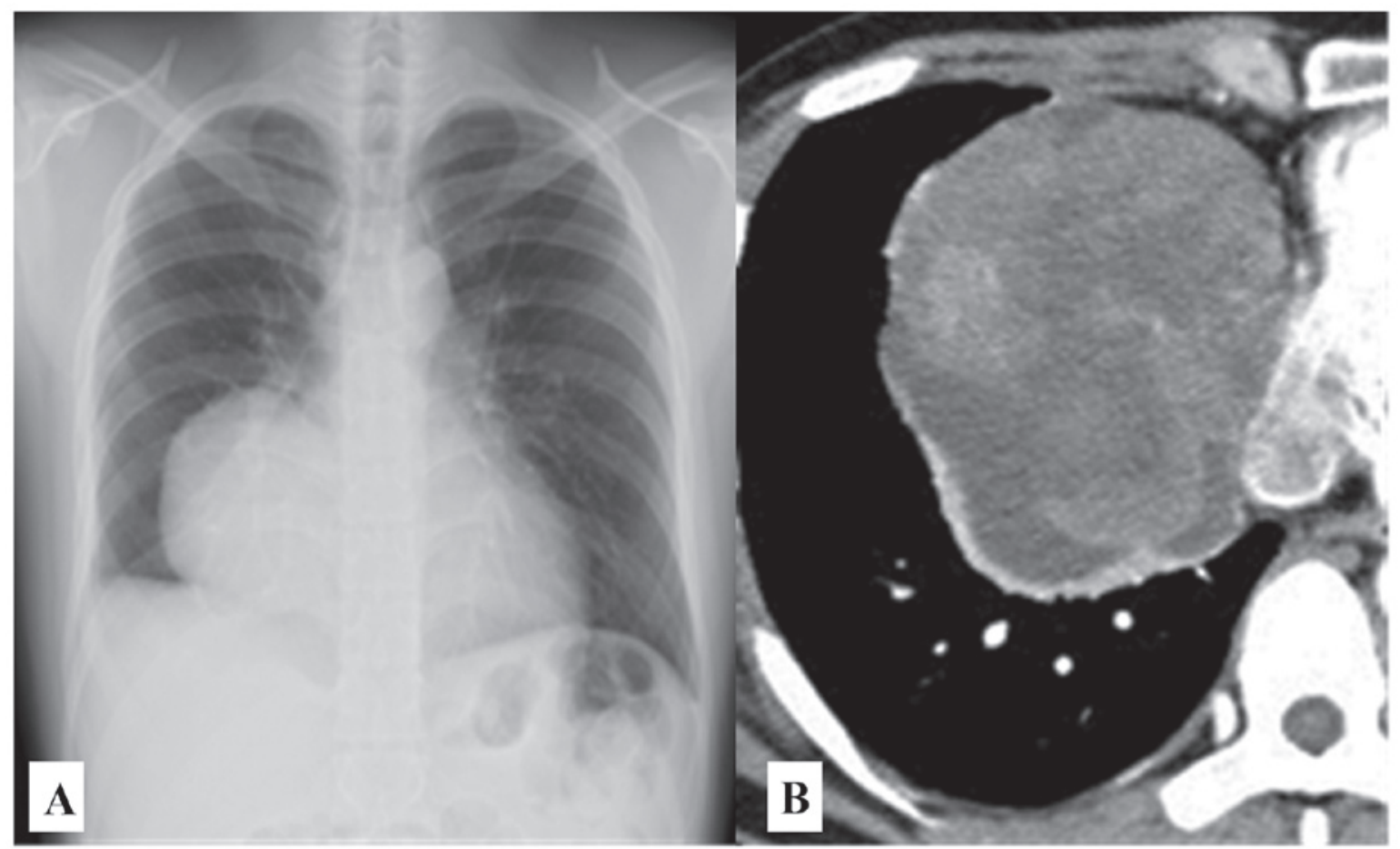

Figure 1. (A) Chest radiography showing a large mass in the right anterior mediastinum. (B) Contrast-enhanced computed tomography showing a heterogeneous mass with rim enhancement in the right anterior mediastinum.

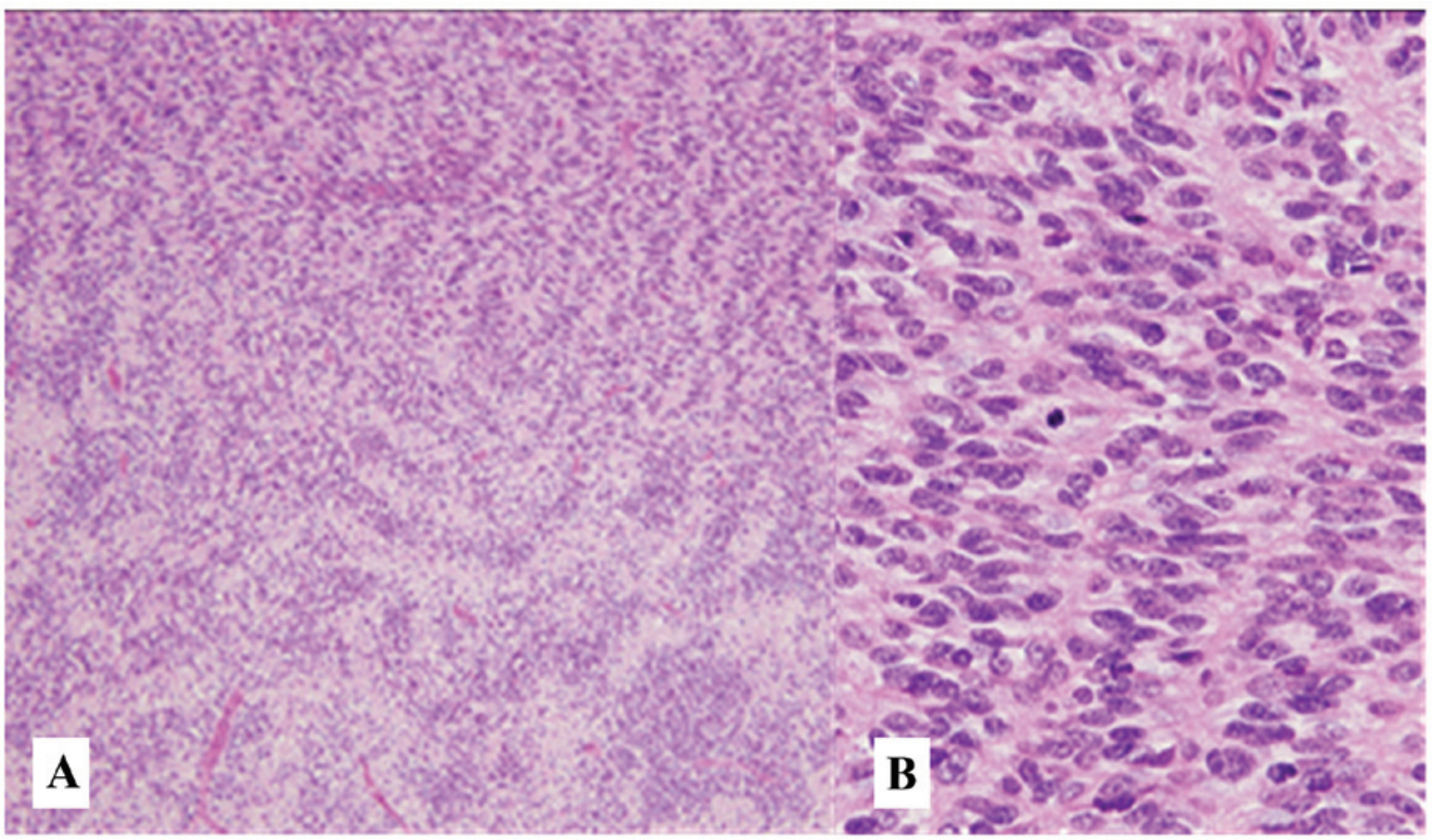

Figure 2. The tumor is composed of highly cellular as well as loose regions containing spindle-shaped tumor cells. Focal nuclear palisading is also observed. Hematoxylin and eosin staining. (A) Magnification, x4; and (B) magnification, x40.

appearance indicating the nervous origin of the tumor (highly cellular and loose regions containing spindle-shaped tumor cells), the diagnosis of MPNST was established.

The postoperative course of the patient was uneventful and there was no need for adjuvant therapy. At 1 year after the surgery, the patient remains free of recurrence.

\section{Discussion}

The majority of mediastinal neurogenic tumors arise in the posterior mediastinum, with only $3 \%$ found in the anterior mediastinum (4). To the best of our knowledge, only 5 patients with MPNST of the anterior mediastinum have been reported 
Table I. Case reports of MPNST in the anterior mediastinum.

\begin{tabular}{|c|c|c|c|c|c|c|c|c|c|}
\hline Author & $\begin{array}{l}\text { Age/ } \\
\text { gender }\end{array}$ & Size, cm & NF1 & Invasion $^{\mathrm{a}}$ & Resection & Recurrence & Metastasis & Outcome & (Refs.) \\
\hline $\begin{array}{l}\text { Ducatman and } \\
\text { Scheithauer }\end{array}$ & $31 / \mathrm{F}$ & Unknown & $(+)$ & Unknown & Incomplete & Unknown & Unknown & DOD at 3 months & $(5)$ \\
\hline Otani et al & $17 / \mathrm{F}$ & Unknown & $(+)$ & $(+)$ & Incomplete & Unknown & $(+)$ & DOD at 7 months & (6) \\
\hline Zisis et al & $30 / \mathrm{M}$ & 6.5 & $(-)$ & $(-)$ & Complete & $(+)$ & $(-)$ & AWD at 1 year & (7) \\
\hline Shimoyama et al & 75/M & 17 & $(-)$ & $(-)$ & Complete & $(-)$ & $(+)$ & DOD at 7 years & (8) \\
\hline Present study & $28 / \mathrm{M}$ & 10 & $(-)$ & $(-)$ & Complete & $(-)$ & $(-)$ & NED at 1 year & \\
\hline
\end{tabular}

${ }^{a}$ Extracapsular tumor invasion. MPNST, malignant peripheral nerve sheath tumor; NF1, neurofibromatosis type 1; F, female; M, male; DOD, dead of disease; AWD, alive with disease; and NED, no evidence of disease.

to date, including our patient (Table I) (5-8). Of all the reported cases, our patient is the only one to have a tumor derived from the phrenic nerve.

The World Health Organization (WHO) classification system defines MPNST as originating from either a peripheral nerve or from a pre-existing benign nerve sheath tumor (usually a neurofibroma), or as occurring in a patient with NF1. The diagnosis is based on a particular constellation of histological and immunohistochemical findings; if these are absent, ultrastructural characteristics suggesting Schwann-cell differentiation are used for diagnosis. According to WHO, MPNST is positive for $\mathrm{S}-100$ in $<50 \%$ of the cases (1). Our patient was negative for S-100; however, we diagnosed the tumor as MPNST since we were able to verify its origin from the right phrenic nerve and since the tumor exhibited the characteristic microscopic features that confirmed its nervous origin.

MPNST arising in the thoracic cavity is difficult to diagnose in its early stages, as the patients are usually asymptomatic. Therefore, tumors of large size, with invasion of the surrounding organs, are occasionally discovered. As MPNST has a very poor prognosis, complete surgical resection is the mainstay of treatment. It is difficult, however, to achieve complete resection with large tumors, as their removal sometimes requires resection of adjacent organs. Of the reported patients with MPNST arising in the anterior mediastinum, complete resection was not achieved in 2 and these patients eventually succumbed to the disease $(5,6)$.

The standard surgical approach for anterior mediastinal tumors is a median sternotomy. However, we performed a posterolateral thoracotomy in our patient in order to gain access to the inferior pulmonary vein. Intraoperatively, the tumor was seen to invade the phrenic nerve and was densely adherent to the middle and lower lobes of the right lung, the pericardium and the diaphragm. We considered it likely that the tumor invaded these surrounding organs and we therefore performed partial resection of these structures. Fortunately, there was no evidence of invasion. Of the 4 priorly reported patients with MPNST arising in the anterior mediastinum, 1 patient exhibited tumor invasion of the surrounding organs, which is associated with a poor prognosis (6).

In a case series reported by Ducatman et al (9), 52\% of the patients with MPSNT had a diagnosis of NF1 and the majority of MPNSTs arose either from neurofibromas or de novo, from normal peripheral nerves. No prior reports were able to identify the nerve origin of the MPNST; however, we were able to confirm that our patient's tumor originated from the right phrenic nerve.

MPNST has a poor prognosis. A case series by Wong et al (10) reported a 5-year survival rate of 52\%, with a $49 \%$ risk of local recurrence and a $49 \%$ risk of distant metastasis. In their retrospective analysis, tumor size, location, patient history of NF1, tumor grade and the integrity of the surgical margins were found to be prognostic factors. Chemotherapy and radiotherapy are often ineffective for patients with MPNST; therefore, the mainstay of treatment is complete surgical resection. However, it was recently demonstrated that adjuvant irradiation is associated with improved local disease control (10). In a series by Carli et al (11), the overall response rate to primary chemotherapy in group 3 patients (using the Intergroup Rhabdomyosarcoma Study grouping system) was $45 \%$ and it was demonstrated that chemotherapy may be effective for patients with unresectable tumors. However, the optimal treatment for MPNST has not been clearly determined and further studies are required to improve the prognosis of patients with MPNST.

In conclusion, our patient presented with an MPNST in the anterior mediastinum originating from the phrenic nerve. Although were able to achieve complete resection, careful follow-up is required for our patient due to the poor prognosis associated with MPNST.

\section{Acknowledgements}

We would like to thank Dr Aki Mitsuda, Department of Surgical Pathology, Toho University, School of Medicine, for her assistance with pathological diagnosis.

\section{References}

1. Nielsen GP, Antonescu CR and Lothe RA: Malignant peripheral nerve sheath tumour. In: WHO Classification of Tumours of Soft Tissue and Bone. Fletcher CDM, Bridge JA, Hogendoorn PCD and Mertens F (eds). Vol 5. 4th edition. IARC Press, Lyon, pp187-189, 2013.

2. Italiano A, Delva F, Mathoulin-Pelissier S, et al: Effect of adjuvant chemotherapy on survival in FNCLCC grade 3 soft tissue sarcomas: a multivariate analysis of the French Sarcoma Group Database. Ann Oncol 21: 2436-2441, 2010. 
3. Sugio K, Inoue T, Inoue K, et al: Neurogenic tumors of the mediastinum originated from the vagus nerve. Eur J Surg Oncol 21: 214-216, 1995

4. Ingels GW, Campbell DC Jr, Giampetro AM, Kozub RE and Bentlage CH: Malignant schwannomas of the mediastinum. Report of two cases and review of the literature. Cancer 27: 1190-1201, 1971.

5. Ducatman BS and Scheithauer BW: Malignant peripheral nerve sheath tumors with divergent differentiation. Cancer 54: 1049-1057, 1984.

6. Otani Y, Morishita Y, Yoshida I, et al: A malignant Triton tumor in the anterior mediastinum requiring emergency surgery: report of a case. Surg Today 26: 834-836, 1996.

7. Zisis C, Fragoulis S, Rontogianni D, Stratakos G and Bellenis I: Malignant triton tumour of the anterior mediastinum as incidental finding. Monaldi Arch Chest Dis 65: 222-224, 2006.
8. Shimoyama T, Yoshida K, Yamoto Y, Koike T and Honma K: Long-term survival after removal of a malignant peripheral nerve sheath tumor originating in the anterior mediastinum. Gen Thorac Cardiovasc Surg 57: 310-314, 2009.

9. Ducatman BS, Scheithauer BW, Piepgras DG, Reiman HM and Ilstrup DM: Malignant peripheral nerve sheath tumors. A clinicopathologic study of 120 cases. Cancer 57: 2006-2021, 1986.

10. Wong WW, Hirose T, Scheithauer BW, Schild SE and Gunderson LL: Malignant peripheral nerve sheath tumor: analysis of treatment outcome. Int J Radiat Oncol Biol Phys 42: 351-360, 1998.

11. Carli M,Ferrari A, Mattke A, et al: Pediatric malignant peripheral nerve sheath tumor: the Italian and German soft tissue sarcoma cooperative group. J Clin Oncol 23: 8422-8430, 2005. 